\title{
Experimental and clinical justification of male orchidectomy under local anesthesia in combination with xylazine and subanesthetic doses of zoletil
}

\author{
Denis Dashko ${ }^{1, *}$, Vyacheslav Tarasevich ${ }^{1}$, and Oleg Melnik ${ }^{2}$ \\ ${ }^{1}$ FSBEI HE "Irkutsk State Agrarian University named after A.A. Ezhevsky", 664038 Irkutsk, Russia \\ ${ }^{2}$ National University of Bioresources and Nature Management of Ukraine, Kiev, Ukraine
}

\begin{abstract}
The objective of our study was to establish the possibility of safe and painless castration of males using only sedatives together with local anesthesia. Animals, before appropriate fixation, were intramuscularly injected with xylanit (active substance xylazine) at a minimum dose of $1 \mathrm{mg} / \mathrm{kg}$ of live weight and zoletil at a subanesthetic dose of $1 \mathrm{mg} / \mathrm{kg}$ of animal weight, the testes and scrotal skin were infiltrated with $2 \%$ lidocaine solution according to the generally accepted method in veterinary surgery. Before, during, and after surgery, nociception indicators were evaluated: blood pressure; heart rate and respiration rate; degree of pain relief depth (presence of pain and spontaneous movement). During the surgery, the heart rate decreased, on average, by $40-50$ beats/minute from baseline values, the respiratory rate decreased, on average, by 10 breaths/minute, and there was a decrease in blood pressure. We found that pre-sedation of animals with xylanite and subanesthetic doses of zoletil followed by tissue infiltration with lidocaine allowed humanely performing orchidectomy, as well as minimizing the cost of general anesthesia and the duration of anesthesia aftereffect on the body.
\end{abstract}

\section{Introduction}

The use of new and effective means, methods and techniques of anesthesia affects many theoretical and practical aspects, both in medicine and in veterinary medicine. Until now, the search for perfect methods of dealing with pain remains an urgent problem in surgery [6]. Domestic and foreign researchers are trying to develop and put into practice new methods of anesthesia, which in their essence would have a number of advantages over chemical means of anesthesia and have the following qualities: safety, dosage accuracy, easy handling, speed of implementation, low degree of toxicity and did not have a negative aftereffect on the body [2, 3, 5-7].

Therefore, the use of inexpensive and high-quality anesthesia in surgery, during various types of surgical interventions in animals is the key to a successful outcome. For example,

\footnotetext{
* Corresponding author: den120577@bk.ru
} 
sedation combined with local anesthesia can be an economical and fast method of performing painful procedures, which is widely used in farm animals in production conditions. Due to the above circumstances, it is necessary to take into account the following fact that when castrating large animals, especially stallions (high differentiation of the central nervous system similar to dogs), only local anesthesia or in combination with "light" sedation is widely used, which in itself does not adversely affect the animal's body and does not complicate the procedure itself. The same principle of "inexpensively qualitatively" can be used in the surgery of small pets, when performing certain operations, such as orchidectomies in dogs, in order to replace general anesthesia (anesthetic condition) with local anesthesia with a combination of subanesthetic doses of narcotic drugs [1]. Local anesthesia with a combination of subanesthetic doses of narcotic drugs for orchidectomy in dogs can also be used when anesthetic equipment is not available or a large number of dogs need to be operated on, which in turn can facilitate the work and reduce the cost of this procedure [10].

In the available literature, we have seen works related to this problem, but in them researchers make ambiguous conclusions about the effectiveness of using local anesthesia in combination with sedatives in some operations in pets. Therefore, in our work, we decided to approach from the perspective of a comprehensive assessment of the effectiveness of anesthesia in males, namely, to register blood pressure indicators (more sensitive indicator of nociception), along with other indicators, before and during the surgery. In addition, we decided to investigate the depth of anesthesia and immobilization in males during orchidectomy, for the first time using the minimum allowable doses of anesthetics and sedatives in the selected combination.

Research objective: a comprehensive experimental and clinical study of the possibility of using local anesthesia in combination with xylazine in minimal doses and zoletil in subanesthetic doses for male orchidectomy.

\section{Material and methods of research}

The research was conducted on the basis of the Irkutsk SAU and veterinary clinics in the city of Irkutsk. The objects of the study were clinically healthy seven males of various breeds aged 7-18 months and weighing 8.7-31.9 kg.

Before, during, and after surgery, the following indicators were evaluated: blood pressure (BP); heart rate (HR) and respiration rate (RR); degree of pain relief depth (presence of pain and spontaneous movement).

Heart and respiration rates, as well as blood pressure, were examined using a Zoomed IM-10 multi-functional veterinary patient monitor. In dogs, these indicators were recorded in the following periods: before the introduction of sedatives; 5 minutes after the introduction of sedatives; before and after the introduction of lidocaine; during skin incision; during the removal of each testicle; during wound suturing; after surgery.

The anesthesia depth was determined by observing the animal's reactions (subjective assessment) during surgery (involuntary movements, sounds, etc.) and evaluated on a scale from 0 to 2 in points: 0 points - absence of any reactions from animal; 1 point - degree of reaction from mild to moderate, which does not interfere with the surgery; 2 points response from animal that makes it difficult or impossible to perform the surgery.

Surgery stages and techniques. Before the surgery, the animals were kept on a 12-hour starvation diet. Dogs were fixed in the dorso-lateral position on the surgery table for small animals. Previously, all animals were sedated with xylanit at a dose of $1 \mathrm{mg} / \mathrm{kg}$ and zoletil 1 $\mathrm{mg} / \mathrm{kg}$ of animal weight in one syringe intramuscularly. After preparation of the surgery field according to the method generally accepted in veterinary surgery, a $2 \%$ lidocaine solution was injected into each testis at the site of the intended incision and intratesticularly. 
Orchidectomy (castration) in males was performed by an open method followed by an intradermal suture on the scrotal surgical wound $[4,8,9]$. To prevent surgical infection, all dogs were intramuscularly injected with a suspension of amoxicillin $15 \%$ at a dose of 1 $\mathrm{ml} / 10 \mathrm{~kg}$ of live weight once after surgery.

For mathematical analysis of the obtained data, the software package "Statistica" was used.

\section{Research results}

During the surgery, the heart rate significantly decreased by an average of 48 beats per minute compared to the baseline level, and the respiratory rate decreased by an average of 9 breaths per minute $(\mathrm{P}<0.05)$ (figure 1$)$.

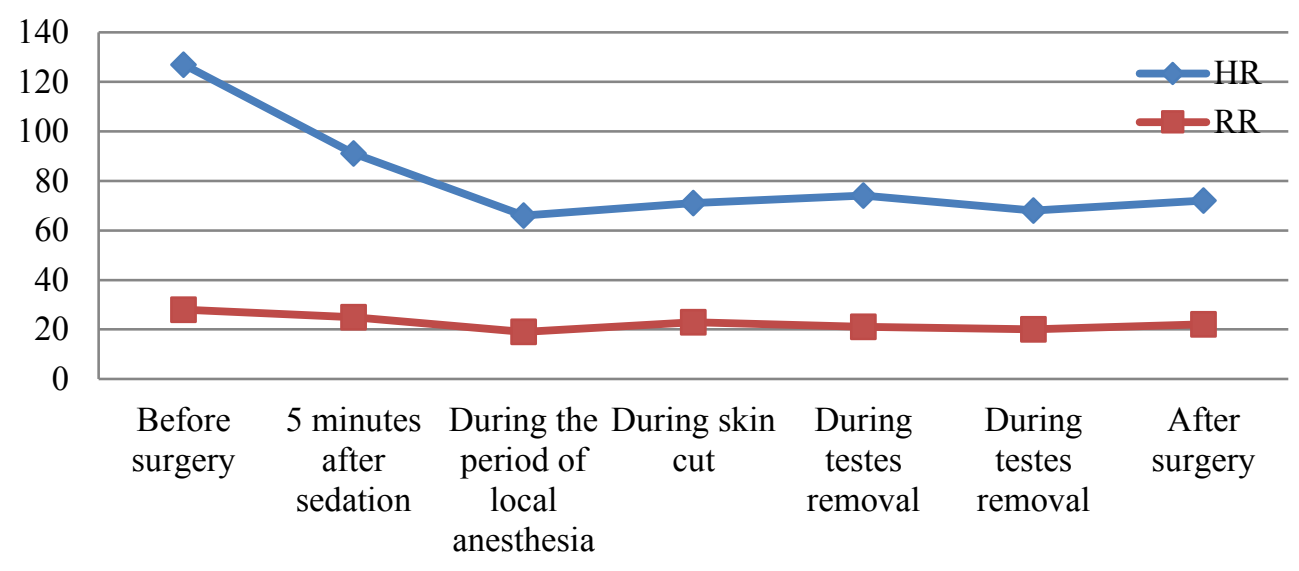

Fig. 1. Heart rate and respiratory rate, per minute.

In animals, there was a tendency to decrease blood pressure, starting from 5 minutes after sedation $(\mathrm{P}<0.05)$ (figure 2). In addition, each studied indicator (heart rate, RR, BP) significantly decreased and reached the minimum value, from the baseline indicators, in dogs during surgery.

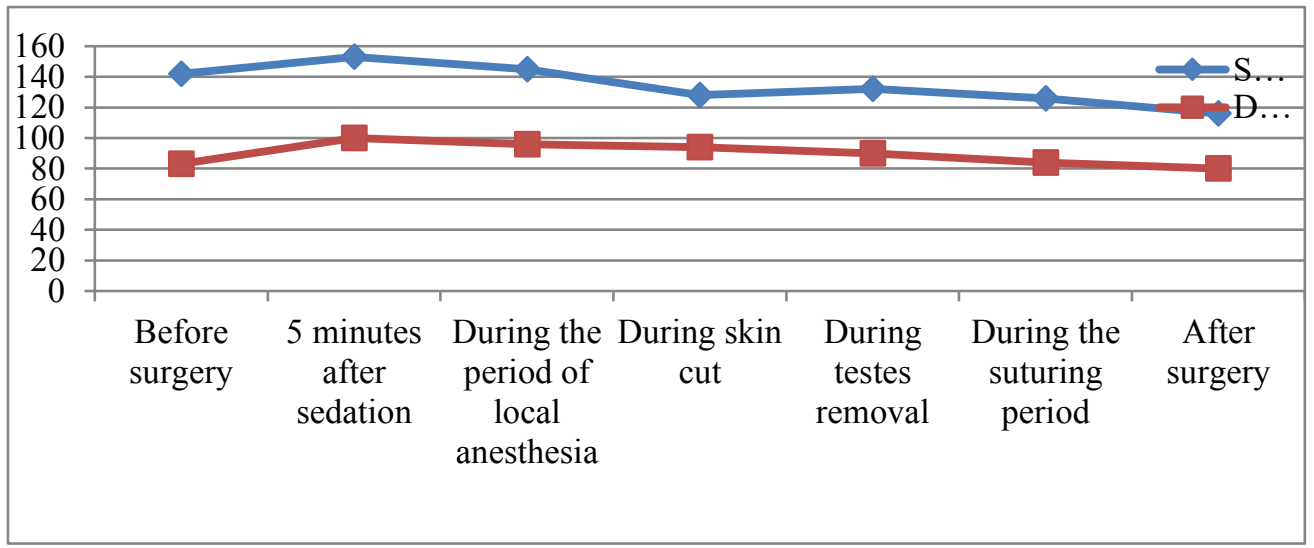

Fig. 2. Systolic and diastolic blood pressure, millimeters of mercury. 
It should be noted that the heart and respiration rates, blood pressure tended to fluctuate throughout the procedure, but significant changes from the baseline were observed during the surgery.

The depth of anesthesia, in the form of spontaneous reactions on the part of the animal during surgery, was significantly lower than before and after the surgery (figure 3 ).

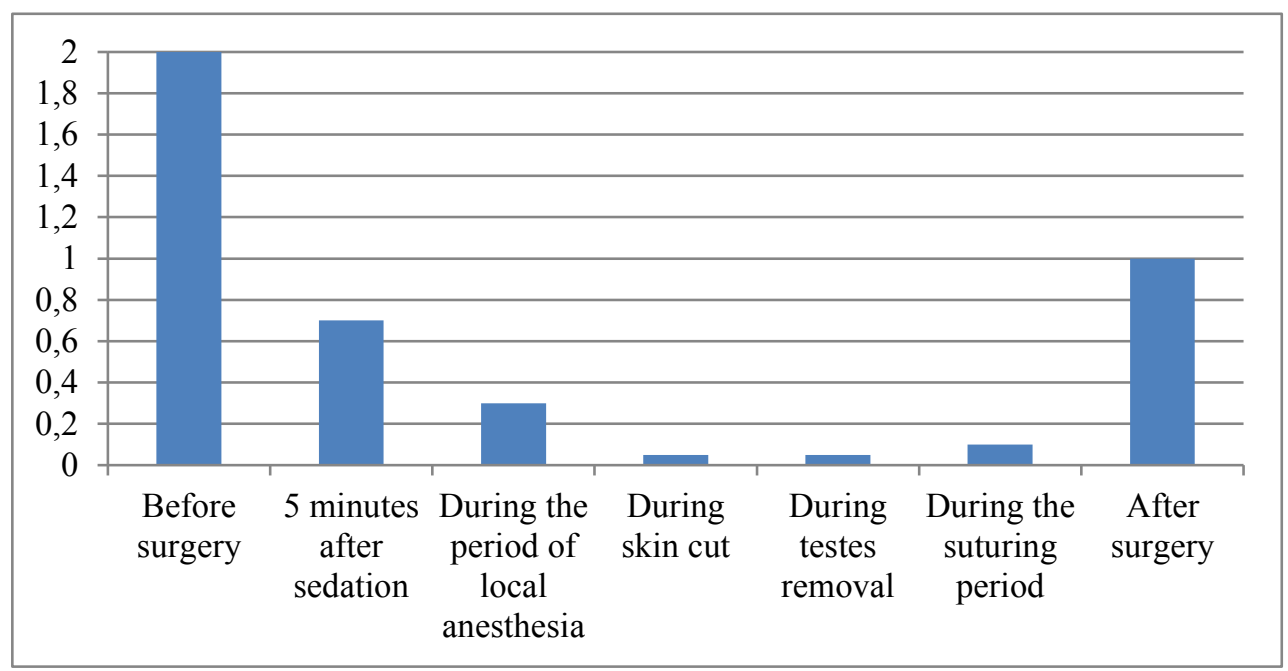

Fig. 3. Anesthesia depth, in points.

During the surgery, the dogs behaved calmly, not responding to pain. In the postsurgical period, there were no complications associated with anesthesia and surgery, except for a slight hyperemia and swelling of the tissues at the site of surgical access.

\section{Conclusion}

Analyzing the data obtained, it should be noted that during the period of surgical intervention, blood pressure, heart rate and respiration rate in males did not increase, but on the contrary, moderately decreased. There was no forced motor response of dogs in response to pain stimuli during surgery. These facts suggest that the depth of anesthesia and immobilization in animals was at the right level for the surgery, because the nociception indicators (BP, HR, RR) did not show vegetative effects (increased heart rate and respiration rate, increased blood pressure, etc.) in response to external pain irritation due to the fact that the pain impulse from the periphery did not reach the spinal cord pathways. Thus, the use of local anesthesia in combination with xylazin in minimal doses and zoletil in subanesthetic doses for male orchidectomy allows humanely castrating dogs and avoiding anesthetic risks and, as a result, reducing the cost of general anesthesia in general.

\section{References}

1. R. Betschart-Wolfensberger, A.A. Stekolnikov, A.Yu. Nechaev, Veterinary anaesthesiology (St. Petersburg: Publishing house "SpetsLit", 2010)

2. A.V. Glotova, Experimental use of electro-anesthesia in dogs, Value of scientific student circles in the innovative development of the agro-industrial complex of the region (Molodezhny: Publishing house of the Irkutsk SAU, 2019) 
3. D.V. Dashko, V.N. Tarasevich, Relevance of the use of transcranial electrical stimulation and electro-anesthesia in veterinary practice, Materials of the VIII International Scientific and Practical Conference "Climate, ecology, agriculture of Eurasia"(Molodezhny: Publishing house of the Irkutsk SAU, 2019)

4. D.V. Dashko, I.I. Silkin, Veterinary surgical terminology (Molodezhny: Publishing house of the Irkutsk SAU, 2020)

5. D.V. Dashko, Eurasian Scientific Association, 3-2(61), 154-156 (2020)

6. D.V. Dashko, Colloquium-journal, 22-2(46), 29-32 (2019)

7. D.V.Dashko, I.I. Silkin, V.N. Tarasevich, Transcranial electroanalgesia and electrical stimulation in veterinary medicine (Molodezhny: Publishing house of the Irkutsk SAU, 2020)

8. A.Yu. Kornilov, Ya.S. Kiseleva, N.V. Gorbunova, Application of the ChassaignacHalsted suture in veterinary surgery, Materials of the All-Russian Scientific and Practical Conference (Molodezhny: Publishing house of the Irkutsk SAU, 2018)

9. K.A. Petrakov, P.T. Salenko, S.M. Paninsky, Operative surgery with topographical anatomy of animals (M.: Koloss, 2003)

10. W. Zink, B.M. Graf, Anesthetist, 52(12), 1102-1123 (2003) 